\title{
RECURRENT METASTASIS OF EPITHELIAL OVARIAN CANCER IN CERVICAL, SUBMANDIBULAR AND SUBOCCIPITAL LYMPH NODES
}

\author{
AZAMSADAT MOUSAVI ${ }^{1}$, FARIBA BEHNAMFAR ${ }^{2}$, NILI MEHRDAD $^{3}$
}

\begin{abstract}
Ovarian carcinoma is the second most common gynecologic cancer and the leading cause of death from gynecologic malignancy. The management of recurrent ovarian cancer has been individualized. Reports of recurrence in supradiaphragmatic lymph nodes are rare. We describe the finding of metastatic tumor involving cervical, submandibular and suboccipital lymph nodes in a 77-year-old patient with ovarian carcinoma.
\end{abstract}

Keywords: scalene lymph node, ovarian cancer, lymphadenopathy, recurrence

\section{Introduction:}

Ovarian carcinoma is the second most common gynecologic cancer and the leading cause of death from gynecologic malignancy. It is generally recognized that ovarian cancer tends to remain intraabdominal even in advanced cases and that dissemination is usually by invasion of adjacent viscera, diffuse intraperitoneal implantation, and metastatic involvement of aortic and pelvic lymph nodes. ${ }^{1}$ Lymphatic dissemination to the pelvic and paraaortic lymph nodes is common in advanced-stage disease. Spread through the lymphatic channels of the diaphragm and through the retroperitoneal lymph nodes can lead to dissemination above the diaphragm, especially the supraclavicular lymph nodes. Extra-abdominal lymph nodes are rarely involved and their detection may represent a challenge for the oncologist. ${ }^{2}$ Few authors have described spread of ovarian cancer to supradiaphragmatic lymph nodes. We describe a patient a with ovarian cancer who had involvement of her scalene lymph nodes several years following her initial diagnosis.

\section{Case report:}

At the time of the original presentation the patient was a 75-year-old female. She presented with the complaint of pelvic pressure and difficult urination. Computed tomography (CT scan) of the abdomen and pelvis revealed a large cystic mass with well defined margins. A total abdominal hysterectomy with bilateral salpingooophorectomy and omentectomy were performed without complication and revealed bilateral moderately differentiated papillary serous cyst adenocarcinoma metastatic to omentum (stage IIIc). There was no bulky retroperitoneal lymph nodes. Postoperatively, the patient received five cycles of carboplatine-paclitaxel chemotherapy. Six cycles were scheduled but the patient did not return to receive the last cycle as instructed. She did well for 25 months, at which time she was found to have unilateral scalene lymph node enlargement. The lymphadenopathy measured $3 \mathrm{X} 3 \mathrm{~cm}$ and was located in left inferior cervical area. Incisional lymphadenopathy biopsy showed metastatic adenocarcinoma. The lesion was unresectable. Computed tomography scan of the thorax, abdomen and pelvis was normal. As a second line therapy she received six courses of carboplatine plus paclitaxel. Lymphadenopathy decreased in size but not disappeared. This was followed up with maintenance therapy of daily oral tamoxifen. After two months taking the tamoxifen maintenance therapy, imaging studies were performed. Abdominopelvic CT scan revealed no abnormalities. But CT of chest showed right paravertebral and precarinal lymphadenopathy [Figure 1]. No pleural effusion or abnormalities in lung parenchyma were found.

1 Gyneco-oncologist, Vali-E-Asr Hospital, Tehran, Iran

2. Fellowship of gyneco-oncology, Vali-E-Asr Hospital, Tehran, Iran

3. Fellowship. of gyneco-oncology, Vali-E-Asr Hospital, Tehran, Iran

Correspondence: Dr. Nili Mehrdad, Fellowship of gyneco-oncology, Vali-E-Asr Hospital, Tehran, Iran. E-mail: nilimehrdad@gmail.com 


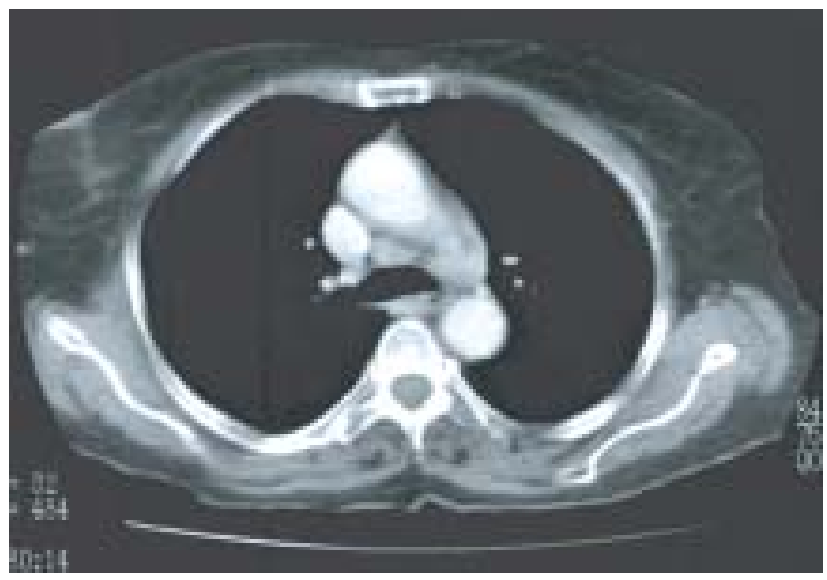

Fig.-1: CT of chest showed right paravertebral and precarinal lymphadenopathy

Four months later, the patient was readmitted with multiple lymphadenopathy involving cervical, suboccipital and submandibular lymph nodes. This time, she received six courses of gemcitabine plus carboplatine and placed on maintenance etoposide. Again lymphadenopathies decreased in size but not disappeared. After two months taking the etopozide maintenance therapy, thoracic CT scan showed bilateral pleural effusion. Abdominopelvic CT scan showed severe left hydronephrosis, marked dilatation of left ureter, small bowel wall thickening and dilatation of adjacent loops. These findings were suggestive of peritoneal seeding or a new metastatic lesion. She died 1 month later with apparent clinical progression of her disease.

\section{Discussion:}

Approximately $80 \%$ of epithelial ovarian carcinomas are discovered as stage III or IV. Histologically, 90\% are epithelial in origin with a small portion being poorly differentiated adenocarcinomas. The mean age of diagnosis is 60 years. ${ }^{3}$ Tumoral dissemination of ovarian cancer most commonly occurs through the intraperitoneal route; nevertheless, although it is rare, ovarian cancer may also metastasize through the lymphatic channels. ${ }^{2}$ Ovarian cancer has three main routes of lymphatic spread .Primary ovarian lymphatic drainage occurs via the infundibulopelvic ligament to the paraaortic nodes. ${ }^{1}$ Lymphatic vessels accompany the ovarian blood vessels cranially to the paraaortic and paracaval lymph nodes. A further route follows the subovarian plexus in the broad ligament to the obturator and pelvic lymph nodes. The third route follows the round ligament to the external iliac and inguinal lymph nodes. ${ }^{4}$ The rate of lymph node metastasis increases by stage. In the literature, the incidence of lymphatic metastasis was $12-25 \%$ in stage I, and 46.9-76.6\% in advanced stages. ${ }^{5}$ In Ayhan's study, lymphatic metastasis was observed in $71.3 \%$ of the patients with stage III-IV disease, while $85.8 \%$ of the patients with stage I-II disease were free of nodal metastasis. ${ }^{5}$ The diagnosis of calcified metastasis of ovarian papillary serous adenocarcinoma can be difficult in the supradiaphragmatic area, where such metastases are unusual. ${ }^{6}$ Petru et al. ${ }^{7}$ reported positive scalene nodes in 7(22\%) of 32 patients with stage III or IV ovarian carcinoma. ${ }^{7}$ Patel et al. reported three case of supradiaphragmatic metatasis, in neck, mediastinal and axillary nodes 3 to 5 years after complete abdomino- pelvic remission. Orris et al. ${ }^{9}$ reported a recurrent ovarian adenocarcinoma in bilateral axillary lymph nodes in a 63-year-old woman. ${ }^{9}$ Gontier et al. reported a 72-year-old woman who had been treated earlier by total abdominal hysterectomy, bilateral salpingo- oophorectomy, and omentectomy, followed by chemotherapy, for an ovarian papillary serous adenocarcinoma. At her annual follow up 16 years later, she presented with a metastatic lesion in the left inferior cervical area. In our presented case, the patient had a shorter diseasefree interval. She first recurred with a single lymphadenopathy in left cervical area. Probably a microscopically abdominal recurrence was also present at the first recurrence although it was not detectable. The rapid progression till death in spite of treatment, suggest it. Later she had multiple lymphadenopathies in cervical, submandibular and suboccipital areas. It has been reported that $75 \%$ of all ovarian cancer patients have a likelihood of disease relapse. Owing to the high probability of relapse, all patients with complete remission of ovarian cancer have to be included in an accurate biochemical and instrumental follow up $\cdot{ }^{10}$ In our patient, cervical lymphadenopathy was the only manifestation of recurrence. These cases show the possibility of apparently isolated supradiaphragmatic relapse, which has been previously described in patients with papillary serous ovarian cancer and which may occur many years after complete remission of the original disease. A careful examination of all lymph node-bearing areas should be done at each follow up visit. In this case, the recurrence was detected clinically. Imaging modalities would allow appropriate therapy to be initiated early during follow up, thus increasing the probability of 
survival. Efficacy of a single PET/CT procedure in detecting recurrence of ovarian cancer has been described, its impact on the clinical management of these patients has not yet been clarified. ${ }^{10}$ A multitude of treatment options are available at the time of recurrence, but there is no clear consensus about how these patients should be managed. Options include surgery, chemotherapy, hormones, and sometimes, radiation therapy. The need for additional effective therapy in most patients is recognized. Though many of recurrences are at distant sites, in a number of patients localized recurrences occur. In our patient, lymphadenopathy was unresectable. We used chemotherapy regimens, however response rate decreases with each subsequent regimen. The issue concerning lymphadenectomy in the management of the disease is still debated. ${ }^{11}$ In patients affected by advanced ovarian cancer, systematic lymphadenectomy statistically significantly improves progression-free survival and reduces recurrence rates despite a higher incidence of postoperative complications. As improvement of overall survival is not statistically significant, further studies are needed to balance risks and benefits of systematic lymphadenectomy in advanced stage disease. ${ }^{12}$ Our patient presented with advanced stage disease and there was no bulky retoperitoneal lymph nodes, so we did not perform lymphadenectomy. The disease recurred in peripheral lymph nodes. Beneditti Panici et al. tried to address the controversial issue of the therapeutic value of lymph node dissection in patients with optimally or completely debulked stage IIIc ovarian carcinoma. They confirmed the high rate of nodal involvement in advanced-stage ovarian cancer ( $70 \%$ of patients in the lymphadenectomy arm versus $42 \%$ in no lymphadenectomy arm). ${ }^{13}$ Given this rate, nodal spread would have been misdiagnosed in approximately half of the patients with nodal metastasis if only bulky nodes had been removed. Such a result is consistent with data in the literature, which show that half of advanced-stage ovarian cancer patients with nodal involvement have no suspicious nodes during macroscopic examination at the time of the surgical procedure. ${ }^{14}$ We believe our patient might belong to this group. Recent series suggest that the aim of debulking surgery is not simply to achieve optimal surgery(i.e., residual disease $<1 \mathrm{~cm}$ ) but rather complete removal of all macroscopic disease. ${ }^{15}$ If a surgical effort is expanded to achieve complete resection of peritoneal disease, this effort should also theoretically include retoperitoneal areas.

\section{Conclusion:}

Ovarian cancer patients have a high likelihood of disease relapse. Owing to the high probability of relapse, all patients with complete remission of ovarian cancer have to be included in an accurate biochemical and instrumental follow up. Careful examination of all lymph node-bearing areas should be done at each follow up visit The observation of the risk of underestimating the tumor spread of advanced ovarian cancer in patients not undergoing systematic lymphadenectomy is certainly an important motivation for performing this procedure.

\section{References:}

1. McGonigle KF, Dudzinski MR. Endometrioid carcinoma of the ovary presenting with an enlarged inguinal lymph node without evidence of abdominal carcinomatosis. Gynecol Oncol 1992; 45(2):225-8.

2. Fanti S, Nanni C, Castellucci P, et, ai. Supraclavicular lymph node metastatic spread in patients with ovarian cancer disclosed at 18F-FDG-PET/CT: an unusual finding. Cancer imaging.2006; 6:20-23.

3. Sher-Ahmed A, Buscema J, Sardi A . A case report of recurrent epithelial ovarian cancer metastatic to the sternum, diaphragm, costae, and bowel managed by aggressive secondary cytoreductive surgery without postoperative chemotherapy. Gynecologic Oncology 2002; 86 (1):91-94.

4. Scholz H.S,Lax S, Tamussino K.F, Petru E . Inguinal lymph node metastasis as only manifestation of lymphatic spread in ovarian cancer: A case report. Gynecologic oncology 1999; 75:517-518.

5. Ayhan A, Gultekin M, Taskiran C, et, al. Lymphatic metastasis in epithelial ovarian carcinoma with respect to clinicopathological variables. Gynecologic Oncology 2005; 97(2):400-404.

6. Gontier E, Wartski M, Guinebretiere J.M, Alberini J.L . F-FDG PET/CT in a patient with lymph node metastasis from ovarian adenocarcinoma. American Journal Of Roentgenology 2006; 187(3): 285-289.

7. Petru E, Pickel H, Tamussino k, et, al. Pretherapeutic scalene lymph node biopsy in ovarian cancer. Gynecologic Oncology 1991; 43(3):262-264.

8. Patel SV, Spencer JA, Wilkinson N, Perren TJ. Supradiaphragmatic manifestations of papillary serous adenocarcinoma of the ovary. Clinical Radiology 1999; 54(11):748-54.

9. Orris BG, Geisler JP, Giesler HE. Ovarian carcinoma metastatic to bilateral axillary lymph nodes. A case report. Eur J Gynaecol Oncol 1999;20(3):189-90. 
10. Mangili G, Picchio M, Sironi S, et, al.. Integrated $\mathrm{PET} / \mathrm{CT}$ as a first-line re-staging modality in patients with suspected recurrence of ovarian cancer. European Journal of Nuclear Medicine and Molecular Imaging 2006 [Epub ahead of print].

11. Angioli R, Plotti F, Palaia I, et, al.Update on lymphadenectomy in early and advanced ovarian cancer. Current Opinion in Obstetrics \& Gynecology. 2008; 20(1):34-39.

12. Ushijima . Management of retroperitoneal lymph nodes in the treatment of ovarian cancer. International Journal of Clinical Oncology 2007; 12(3):181-86.
13. Panici PB, Maggioni A, Hacker N, et, al. Systematic aortic and pelvic lymphadenectomy versus resection of bulky nodes only in optimally debulked advanced ovarian cancer: a randomized clinical trial. Journal of National cancer institue 2005; 97(8):560-66.

14. Morice P, Joulie F, Camatte S, et, al.Lymph node involvement in epithelial ovarian cancer: analysis of 276 pelvic and paraaortic lymphadenectomies and surgical implications. Journal of American College of Surgery 2003;197(2): 198-205.

15. Chi DS, Eisenhauer EL, Lang J, et, al. What is the optimal goal of primary cytoreductive surgery for bulky stage IIIC epithelial ovarian carcinoma (EOC)?. Gynecology Oncology 2006;103(2):559-64. 